\title{
Realization of a magnetically guided atomic beam in the collisional regime
}

\author{
T. Lahaye, J. M. Vogels, K. Günter, Z. Wang, J. Dalibard, and D. Guéry-Odelin \\ Laboratoire Kastler Brossel*, 24 rue Lhomond, F-75231 Paris Cedex 05, France
}

(Dated: November 14, 2018)

\begin{abstract}
We describe the realization of a magnetically guided beam of cold rubidium atoms, with a flux of $7 \times 10^{9}$ atoms/s, a temperature of $400 \mu \mathrm{K}$ and a mean velocity of $1 \mathrm{~m} / \mathrm{s}$. The rate of elastic collisions within the beam is sufficient to ensure thermalization. We show that the evaporation induced by a radio-frequency wave leads to appreciable cooling and increase in phase space density. We discuss the perspectives to reach the quantum degenerate regime using evaporative cooling.

PACS numbers: $32.80 . \mathrm{Pj}, 03.75 . \mathrm{Pp}$
\end{abstract}

The recent realization of slow atom sources using laser cooling methods has constituted a major advance for many applications in metrology and matter wave interferometry [1]. The possibility to complement laser cooling by forced evaporation has led to even colder and denser atomic gases, and has culminated with the observation of Bose-Einstein condensation (BEC) 2] and pulsed "atom lasers" extracted from the condensate [3]. These advances in atom cooling allow in principle to build a continuous and coherent source of matter waves which would be the equivalent of a monochromatic laser. It should lead to unprecedented performances in terms of focalization or collimation, and provide a unique tool for future developments in atom manipulation and precision measurements.

Two paths have been considered to reach the goal of a continuous and coherent source of atoms ("cw atom laser"). The first possibility [4] consists in using the "standard" condensation procedure to periodically replenish with new condensates a gas sample held in an optical dipole trap. The second possibility, that we are currently investigating, consists in transposing the evaporative cooling method to a guided atomic beam [5]. As the beam progresses in a magnetic guide [ 6 , 7], the most energetic atoms are removed and the remaining particles thermalize at a lower temperature and a larger density, so that the beam can eventually reach the degenerate regime. This method should in principle allow for a large flux, since it consists in a parallel implementation of the various steps to BEC. Instead of performing sequentially the optical cooling and the evaporation sequences at the same place, these occur simultaneously in different locations along the guided beam. This gain in flux can constitute a major advantage for applications.

To implement this method, the initial atomic beam needs to be deeply in the collisional regime. The number $N_{\text {col }}$ of elastic collisions undergone by each atom as it travels along the guide must be large compared to unity, to provide the thermalization required for evaporative cooling. Such beams were so far not available. In this Letter we report on the realization of a rubidium atomic beam in the collisional regime, with $N_{\text {col }} \sim 8$. It propagates in a $4.5 \mathrm{~m}$ long magnetic guide with a mean velocity of $1 \mathrm{~m} / \mathrm{s}$. We also demonstrate a first step of evaporative cooling, and show that the measured increase in phase space density is in good agreement with expectations. Finally we discuss possible improvements of the current setup which should allow to reach the degenerate regime.

Fig. 1 shows the layout of our experimental setup, composed of three main parts lying in the $x y$ horizontal plane: the Zeeman slower along the $y$ axis, the magnetooptical trap (MOT) and the magnetic guide along the $x$ axis. The Zeeman slower [8] consists in a $1.1 \mathrm{~m}$ long tapered solenoid in which atoms emerging from an oven are decelerated by resonant radiation pressure. It delivers a flux of $2 \times 10^{11} \mathrm{~s}^{-1}{ }^{87} \mathrm{Rb}$ atoms, with a velocity of $20 \mathrm{~m} / \mathrm{s}$.

The slow atoms are captured in a 3 -dimensional MOT formed by 3 pairs of counter-propagating beams. One pair of beam is aligned with the vertical $(z)$ axis, and the two other pairs are along the directions $\boldsymbol{e}_{x} \pm \boldsymbol{e}_{y}$. Each trapping beam has a circular profile, with a $1 / e^{2}$ radius of $17 \mathrm{~mm}$ and a power of $15 \mathrm{~mW}$. It is spatially filtered using a single mode optical fiber and intensity stabilized to better than $1 \%$. The MOT magnetic gradients are $b_{x}^{\prime}=0.6 \mathrm{G} / \mathrm{cm}, b_{y}^{\prime}=5.4 \mathrm{G} / \mathrm{cm}$ and $b_{z}^{\prime}=-6 \mathrm{G} / \mathrm{cm}$. The MOT is thus cigar-shaped, and its long axis coincides with the magnetic guide direction. The capture rate of the MOT is $2 \times 10^{10}$ atoms $/ \mathrm{s}$.

The entrance of the magnetic guide is located $4.6 \mathrm{~cm}$ away from the MOT center. The guide, which is placed inside the vacuum system, consists of four parallel, watercooled copper tubes, in which we run a current $I$ up to 400 A. Unless otherwise stated, parameters and results given below correspond to $I=240 \mathrm{~A}$. The entrance of the guide consists in a ring shaped metal part, that is used for the recombination of the electric and cooling water circuits [7]. The guide is held in place and shaped by means of ceramic pieces. At the entrance of the guide the distance between the centers of adjacent tubes is $14 \mathrm{~mm}$, resulting in a gradient of $b_{0}^{\prime}=200 \mathrm{G} / \mathrm{cm}$. The first $40 \mathrm{~cm}$ of the magnetic guide form a tapered section, obtained by reducing progressively the distance between the tubes to $8 \mathrm{~mm}$. This results in an increase of the trapping gradient to $b_{1}^{\prime}=600 \mathrm{G} / \mathrm{cm}$. The guide provides a confinement of the atoms with a linear potential $\mu b^{\prime}\left(y^{2}+z^{2}\right)^{1 / 2}$, where $\mu$ is the magnetic moment of the atoms $[9]$. 


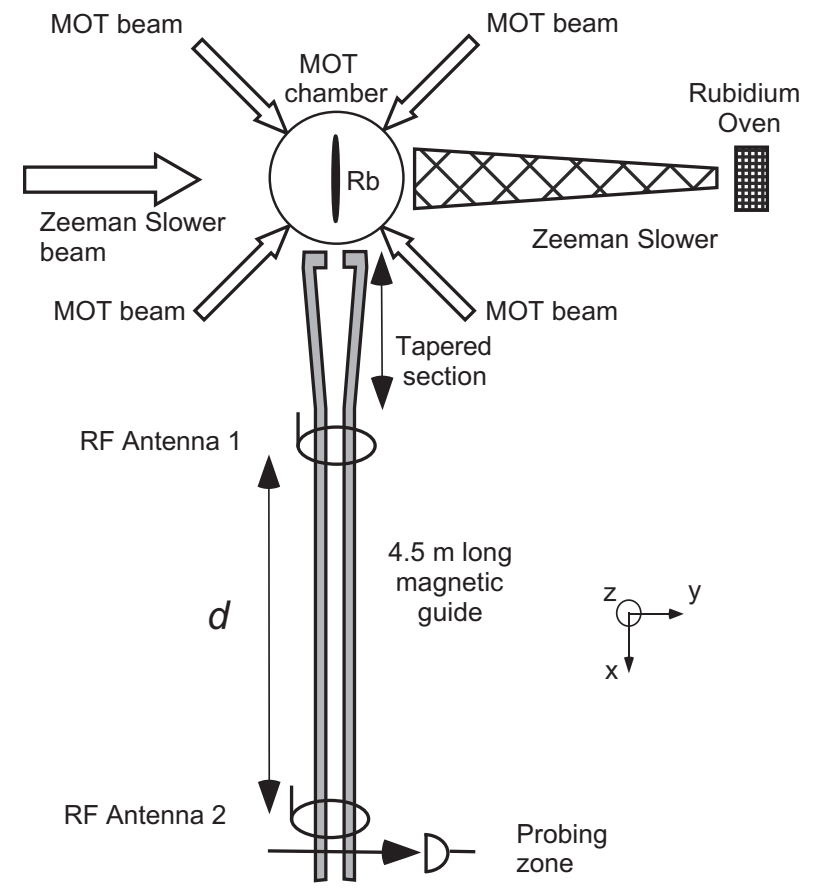

FIG. 1: Top view of the setup. The atoms emerging from the oven are slowed down by radiation pressure, captured in the magneto-optical trap, and launched into the magnetic guide.

To optimize the transfer of the atoms from the MOT into the magnetic guide, we operate in pulsed mode [10], with a duration of $t_{\mathrm{seq}}=283 \mathrm{~ms}$ per sequence. In the first $140 \mathrm{~ms}$ we capture $3 \times 10^{9}$ atoms in the MOT. All beams are set at the same frequency $\omega_{L}$, corresponding to a detuning $\Delta=\omega_{L}-\omega_{A}=-3 \Gamma$, where $\Gamma / 2 \pi=5.9 \mathrm{MHz}$ is the natural width of the $5 P_{3 / 2}$ excited state and $\omega_{A}$ the resonance frequency of a rubidium atom. The trapped cloud is cigar-shaped, with a $1 / \sqrt{e}$ radius of $1.4 \mathrm{~mm}$ and a length of $35 \mathrm{~mm}$.

The MOT magnetic field is then turned off and a $3 \mathrm{~ms}$ launching phase occurs. The detunings of the beams in the $x y$ plane are shifted by $\pm k_{L} v / \sqrt{2}$ with respect to the detuning $\Delta$ of the vertical beams, where $k_{L}=\omega_{L} / c$. This forms a moving molasses which sets the atoms in motion at velocity $v=1.1 \mathrm{~m} / \mathrm{s}$ in the direction of the magnetic guide 11]. The detuning $\Delta$ during the launching phase is ramped to $-10 \Gamma$, which reduces the temperature of the gas to $T_{0}=40 \mu \mathrm{K}$ in the frame moving at velocity $v$.

The final phase of the sequence consists in (i) optical pumping of the moving atoms into the weak-fieldseeking ground state $\left|F=1, m_{F}=-1\right\rangle$, and (ii) magnetic pre-guiding of the atom cloud. The pre-guiding is provided by a two-dimensional $(y z)$ quadrupole magnetic field $b_{2}^{\prime}=65 \mathrm{G} / \mathrm{cm}$ generated by four elongated coils (not shown in Fig. 1), located around the MOT chamber. It prevents the atom cloud from expanding during its flight towards the entrance of the magnetic guide. During the pre-guiding phase we also apply at the MOT location a
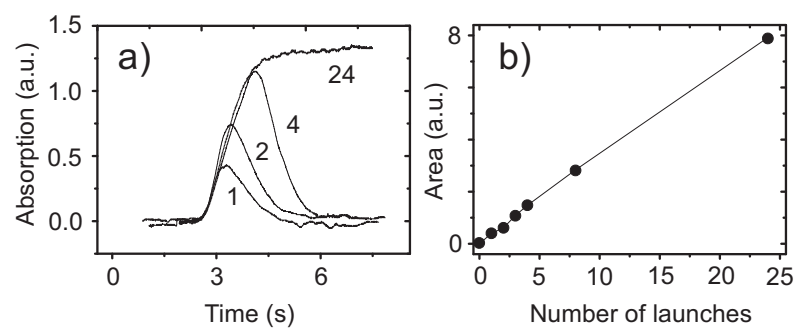

FIG. 2: a) Absorption signal measured at the end of the guide for 1, 2, 4 and 24 launches. b) Number of atoms (area of the absorption signal) as a function of the number of launches. The time origin corresponds to the launching of the first packet.

$56 \mathrm{G}$ bias field along the $x$ axis to match the oscillation frequency in the pre-guide with the cloud parameters. The overall efficiency of the pumping+pre-guiding process is $60 \%$ so that each packet loaded into the guide contains $N_{\text {load }} \sim 2 \times 10^{9}$ atoms. We measure the atom number and mean velocity by monitoring the absorption of a resonant probe beam at the exit of the $4.5 \mathrm{~m}$ magnetic guide. Losses due to collisions with residual gas are negligible.

We have estimated numerically the influence of the rapid variation of the magnetic gradient from $b_{2}^{\prime}$ to $b_{0}^{\prime}$ at the entrance in the main guide by analyzing single particle trajectories. The cloud is compressed to a final transverse radius $r_{0}=400 \mu \mathrm{m}$ and an energy per atom of $k_{B} \times 500 \mu \mathrm{K}$ in the moving frame. In the subsequent propagation into the tapered section of the guide, the gradient increases to $b_{1}^{\prime}$ and the bias field decreases to 0 [9]. It leads to a further, quasi-adiabatic, compression of the cloud and the energy per atom raises to $k_{B} \times 1400 \mu \mathrm{K}$. Assuming that thermalization occurs via elastic collisions, this corresponds to a temperature of $T_{1}=400 \mu \mathrm{K}$ shared as $3 k_{B} T_{1} / 2$ for the kinetic energy in the moving frame and $2 k_{B} T_{1}$ for the potential energy of the transverse linear confinement. This estimate for $T_{1}$ is in good agreement with our measurement as we shall see below.

The next capture+launch sequence starts $140 \mathrm{~ms}$ after the beginning of the pre-guiding phase, so that the atom packet has moved $\sim 14 \mathrm{~cm}$ away from the MOT center and is now well inside the main guide. A remarkable feature of this protocol is that the light scattered from the MOT during the preparation of a packet does not produce detectable losses on the previous packet. Fig. 2 shows the signal detected for different numbers of launches. One can check that the area of the signal scales linearly with this number.

To generate a continuous beam we repeat the sequence with a cycling rate of $t_{\mathrm{seq}}^{-1}=3.5 \mathrm{~Hz}$. For $T=400 \mu \mathrm{K}$ the packets overlap after a propagation of $60 \mathrm{~cm}$. The flux is $\phi=N_{\text {load }} t_{\text {seq }}^{-1}=7( \pm 2) \times 10^{9}$ atoms $/ \mathrm{s}$.

We now turn to the characterization of the beam and the demonstration that the collisional regime has indeed 


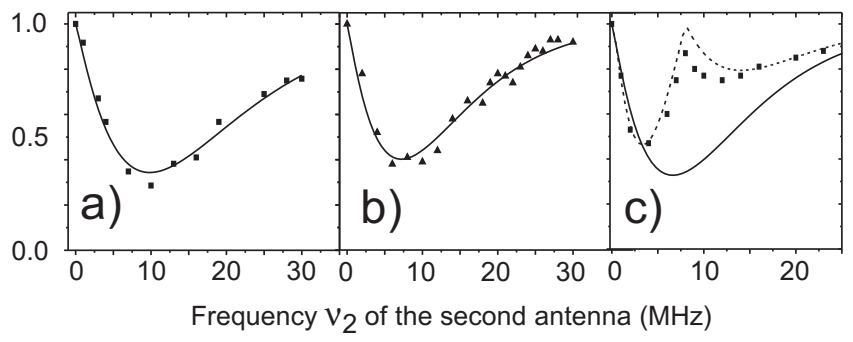

FIG. 3: Experiment with two antennas separated by a distance $d$ : remaining fraction after the second antenna, with the first antenna set at a fixed value $\nu_{1}$. a) Antenna 1 off, flux $\phi, b_{1}^{\prime}=600 \mathrm{G} / \mathrm{cm}$ and $d=3 \mathrm{~m}$. Using (2) as a fit function (solid line), we find $T_{1}=384( \pm 13) \mu \mathrm{K}$. b) $\nu_{1}=24 \mathrm{MHz}$ with the same conditions as for a). The fit gives $T_{2}=281( \pm 8) \mu \mathrm{K}$ indicating a significant cooling. c) $\nu_{1}=8 \mathrm{MHz}$ and reduced number of collisions between antennas: flux $\phi / 3, b_{1}^{\prime}=400 \mathrm{G} / \mathrm{cm}, d=0.85 \mathrm{~m}$. The dotted line is the prediction for the collisionless regime. The solid line is the result expected if thermalization had occurred.

been reached. The main tools for this study are radiofrequency (r.f.) antennas located along the guide, which generate an oscillating magnetic field at frequency $\nu$. An antenna flips the magnetic moment of the atoms passing at a distance $r_{\mathrm{e}}=h \nu /\left(\mu b^{\prime}\right)$ from the $x$ axis (r.f. evaporation [12]). For an atom with transverse energy $E$ and angular momentum $L$ along the $x$ axis, the evaporation criterion $r=r_{\mathrm{e}}$ is fulfilled at some points of the atom trajectory if

$$
E \geq \frac{L^{2}}{2 m r_{\mathrm{e}}^{2}}+\mu b^{\prime} r_{\mathrm{e}}
$$

where $m$ is the atom mass. The range of an antenna is $\sim 20 \mathrm{~cm}$, so that it constitutes a local probe of the $(E, L)$ distribution at the scale of the whole guide.

The temperature in the guide is deduced from the variation of the fraction $f(\nu)$ of remaining atoms after the antenna. For $r_{\mathrm{e}}$ small compared to the thermal size $r_{\text {th }}=2 k_{B} T /\left(\mu b^{\prime}\right)$, only a very small fraction of atoms has a sufficiently low angular momentum to be evaporated, as a consequence $f(\nu \rightarrow 0)=1$. In the opposite limit $r_{\mathrm{e}} \gg r_{\mathrm{th}}$, the evaporated fraction is negligible because of the exponential decay of the energy distribution, thus $f(\infty)=1$. We have used a Monte-Carlo determination of $f(\nu)$, that is accurately fitted by the analytical form:

$$
f(\nu)=1-A \eta^{B} e^{-C \eta} \quad \text { with } \quad \eta=h \nu /\left(k_{B} T\right)
$$

with $A=1.65, B=1.13$ and $C=0.92$. A minimum value $f^{*} \simeq 0.33$ is expected for $h \nu^{*} /\left(k_{B} T\right) \simeq 1.25$.

A typical measurement of $f(\nu)$ is given in Fig. 3a, together with a fit using (2). The transverse temperature corresponding to the best fit is $384( \pm 13) \mu \mathrm{K}$, in good agreement with the expected temperature given above. For the fit we allow the coefficient $A$ in (2) to vary, to

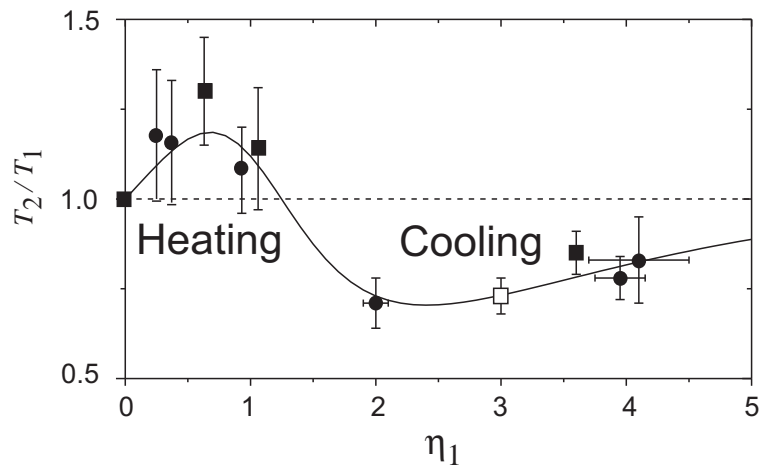

FIG. 4: Ratio $T_{2} / T_{1}$ between the temperature $T_{2}$ of the beam when the first antenna is set at $\nu_{1}$ and the temperature $T_{1}$ of the beam in absence of evaporation, as a function of the dimensionless parameter $\eta_{1}=h \nu_{1} /\left(k_{B} T_{1}\right)$. The solid line is the theoretical prediction for a linear potential (no adjustable parameter). The open square corresponds to the data of Fig. 3b. The squares (resp. circles) have been obtained with $I=$ 240 A (resp. 400 A).

account for a possible uncomplete efficiency of the evaporation process. For the data of Fig. 3a, the efficiency is better than $90 \%$.

The thermal equilibrium assumption used above is valid only if a sufficient number of elastic collisions (at least a few per atom) has occurred in the guide. The good agreement between the shapes of the experimental and predicted functions $f(\nu)$ is a first indication that the collisional regime has indeed been reached. To investigate this point further, we now turn to a twoantenna experiment (Fig. 3b). These antennas are located respectively $d_{1}=1 \mathrm{~m}$ and $d_{2}=4 \mathrm{~m}$ after the entrance of the guide. The frequency $\nu_{1}$ of the first antenna is fixed at $\nu_{1}=24 \mathrm{MHz}$, which corresponds to $\eta_{1} \equiv h \nu_{1} /\left(k_{B} T_{1}\right)=3$, where $T_{1}$ is the temperature deduced from the analysis of Fig. [3. This antenna evaporates the atoms fulfilling (11), thus producing a non thermal $(E, L)$ distribution. We measure the function $f\left(\nu_{2}\right)$ with the second antenna, and check whether a thermal distribution is recovered during the propagation from $d_{1}$ to $d_{2}$. The result for $f\left(\nu_{2}\right)$ in Fig. 3b shows clearly that this is indeed the case. We infer from this set of data a temperature $T_{2}=281( \pm 8) \mu \mathrm{K}$. The fact that $T_{2}<T_{1}$ is a clear demonstration of evaporative cooling of the beam by the first antenna.

We have investigated the thermalization process as a function of the evaporation frequency $\nu_{1}$ (Fig. 4). For $\eta_{1} \lesssim 1.25$ the evaporated atoms have less energy than average, leading to heating $\left(T_{2}>T_{1}\right)$. Cooling is observed in the opposite case $\eta_{1} \gtrsim 1.25$. The continuous line in Fig. 4is the theoretical prediction assuming full thermalization between $d_{1}$ and $d_{2}$. It is in excellent agreement with the experimental results. The phase space density at the center of the beam scales as $\phi / T^{7 / 2}$. When we choose $\eta_{1}=3$ (Fig. 3b), we measure a decrease of the 
flux by $36 \%$ and a decrease of temperature of $27 \%$. This corresponds to an increase in phase space by a factor $1.92( \pm 0.15)$, to be compared with the expected gain of 1.90. It shows that this transverse evaporation mechanism is indeed well suited for our final goal, i.e. bringing the beam in the degenerate regime.

We have also investigated the transition between the collisional and collisionless regimes. For this study we have reduced the collision rate by decreasing the flux to $\phi / 3$ and by operating the magnetic guide at a lower current $(160 \mathrm{~A})$. The measured temperature is in this case $T_{1}=230( \pm 6) \mu \mathrm{K}$. To minimize the thermalization process between the antennas, we have also reduced the distance $d_{2}-d_{1}$ to $0.85 \mathrm{~m}$. The result for $f\left(\nu_{2}\right)$ is shown in Fig. 3, for the particular choice $\nu_{1}=8 \mathrm{MHz}$. The dashed line gives the result expected in the collisionless regime. In this case the second antenna does not induce extra loss when $\nu_{2}=\nu_{1}$ since no modification of the $(E, L)$ distribution occurs between the two antennas. The measured $f\left(\nu_{2}\right)$ is in good agreement with this prediction. For comparison we also indicate in Fig. 3r the expected signal in case of a complete thermalization between the antennas. It clearly does not agree with our data, contrary to the large flux case (Fig. [3p).

To summarize, our guided beam is deeply in the collisional regime when operated at the maximum flux. The collision rate in the linear guide is $\gamma_{c}=n_{0} \sigma \Delta v / \sqrt{\pi}=$ $2 \mathrm{~s}^{-1}$, where $n_{0}=2.8 \times 10^{10} \mathrm{~cm}^{-3}$ is the central density, $\sigma=6.8 \times 10^{-12} \mathrm{~cm}^{2}$ is the s-wave elastic crosssection at low temperature for spin polarized ${ }^{87} \mathrm{Rb}$ atoms and $\Delta v=\sqrt{k_{B} T / m}=19 \mathrm{~cm} / \mathrm{s}$. This corresponds to $N_{\text {col }}=8$ collisions per atom along the whole guide. For the thermalization experiment of Fig. [3] and taking into account the reduction of flux by the first antenna, we estimate that each atom undergoes 3 collisions between the two antennas. This is known to be the minimum value to ensure thermalization (see e.g. [13]).

Finally we briefly discuss the possibility to use this device for producing a coherent continuous atomic wave. The current phase space density at the center of the beam is $2 \times 10^{-8}$. To reach quantum degeneracy, we plan to use several antennas with decreasing frequencies. Operating all antennas at the same $\eta$, the cooling distance is minimized for $\eta \sim 4$. A total of 40 antennas (each providing a phase space increase of 1.63) is needed. The expected output flux is $2 \times 10^{-4} \phi$ for a cooling distance of $\sim 100 \mathrm{v} / \gamma_{c}$. To match this distance with the length of our guide, we need to increase the initial collision rate $\gamma_{c}$ by one order of magnitude. A fraction of this gain can be obtained by slowing down the beam by gravity, using a bent or tilted guide 14. In addition we plan to transpose to our elongated MOT the "dark spot" technique, which is known to lead to a significant gain in terms of spatial density of the laser cooled atomic source [15]. A continuous coherent atomic beam will be a very useful tool not only for the interferometric and metrol- ogy applications mentioned above, but also for studies of fundamental issues, such as the existence of superfluidity in quasi one-dimensional systems. Another exciting challenge will be the miniaturization of the setup, along the line of what has been achieved recently with "atom chips" for the production of standard BEC [16].

We acknowledge stimulating discussion with the ENS laser cooling group. We thank A. Senger for his participation to the data acquisition. K. Günter acknowledges support from the Marie Curie Host Fellowship "QPAF" HPMT-CT-2000-00102. J. M. Vogels acknowledges support from the Research Training Network "Cold Quantum Gases" HPRN-CT-2000-00125. This work was partially supported by the CNRS, the Région Ile-de-France, the Collège de France, the Ecole Normale Supérieure, the Bureau National de la Métrologie, the University of Paris VI and the Délégation Générale de l'Armement.

[*] Unité de Recherche de l'Ecole Normale Supérieure et de l'Université Pierre et Marie Curie, associée au CNRS.

[1] P. R. Berman, Atom interferometry, (Academic Press, London, 1997).

[2] C. J. Pethick and H. Smith, Bose-Einstein Condensation in Dilute Gases, (Cambridge University Press, Cambridge, 2002); L. P. Pitaevskii and S. Stringari, BoseEinstein condensation, (Clarendon Press, Oxford, 2003).

[3] K. Helmerson, D. Hutchinson and W. Phillips, Physics World 12, 31 (1999) and refs. therein.

[4] A. P. Chikkatur et al., Science 296, 2193 (2002).

[5] E. Mandonnet et al., Eur. Phys J. D 10, 9 (2000).

[6] M. Key et al., Phys. Rev. Lett. 84, 1371 (2000); N. H. Dekker et al., Phys. Rev. Lett. 84, 1124 (2000); B. K. Teo and G. Raithel, Phys. Rev. A 63, 031402 (2001); J. A. Sauer, M. D. Barrett, and M. S. Chapman, Phys. Rev. Lett. 87, 270401 (2001).

[7] P. Cren et al., Eur. Phys J. D 20, 107 (2002).

[8] H. J. Metcalf and P. van der Straten, Laser Cooling and Trapping, (Springer-Verlag, New York, 1999).

[9] Strictly speaking, the magnetic field at the center of the guide does not vanish thanks to the $x$ component of the Earth magnetic field, which prevents Majorana spin flips.

[10] J. M. Vogels et al., cond-mat/0404560

[11] A. Clairon, C. Salomon, S. Guellati. and W.D. Phillips, Europhys. Lett., 16, 165 (1991).

[12] O. Luiten, M. Reynolds, J. Walraven, Phys. Rev. A 53, 381 (1996); W. Ketterle and N. van Druten, Adv. At. Mol. Opt. Phys. 37, 181 (1996).

[13] C. R. Monroe et al., Phys. Rev. Lett. 70, 414, (1993).

[14] T. Lahaye, P. Cren, C. Roos, D. Guéry-Odelin, Comm. Nonlin. Sci. Num. Sim. 8, 315 (2003).

[15] W. Ketterle et al., Phys. Rev. Lett. 70, 2253, (1993).

[16] W. Hänsel, P. Hommelhoff, T. W. Hänsch, and J. Reichel, Nature (London) 413, 498 (2001); H. Ott, J. Fortägh, G. Schlotterbeck, A. Grossmann, and C. Zimmermann, Phys. Rev. Lett. 87, 230401 (2001); A. E. Leanhardt et al., ibid 89, 040401 (2002); S. Schneider et al. Phys. Rev. A 67, 023612 (2003). 